\title{
Qualitätssicherung in der Bauteilreinigung
}

\author{
Ein Grundlagenseminar zeigt in Theorie und Praxis auf, wie Reinigungsprozesse bedarfsgerecht \\ ausgelegt beziehungsweise optimiert, gesteuert und überwacht werden können.
}

Als Querschnittsthema hat die Bauteilreinigung eine hohe Relevanz in allen Fertigungsbranchen. Da reinigungstechnisches Wissen bisher jedoch kaum in der Ausbildung beziehungsweise im Studium vermittelt wird, bestehen in diesem Bereich der Fertigung häufig noch Lücken.

Um diese Lücken zu schließen, hat der Fachverband industrielle Teilereinigung e.V. (FiT) das Grundlagenseminar „Qua-

litätssicherung in der Bauteilreinigung“ erarbeitet. Das von fairXperts durchgeführte Seminar besteht aus zwei Veranstaltungen, die unabhängig voneinander besucht werden können. In Theorie und Praxis erhalten die Teilnehmer dabei das notwendige Wissen und die Qualifikation, um Reinigungsprozesse abgestimmt auf Bauteilmaterial, -geometrie und -verunreinigung sowie angepasst an die jeweili-

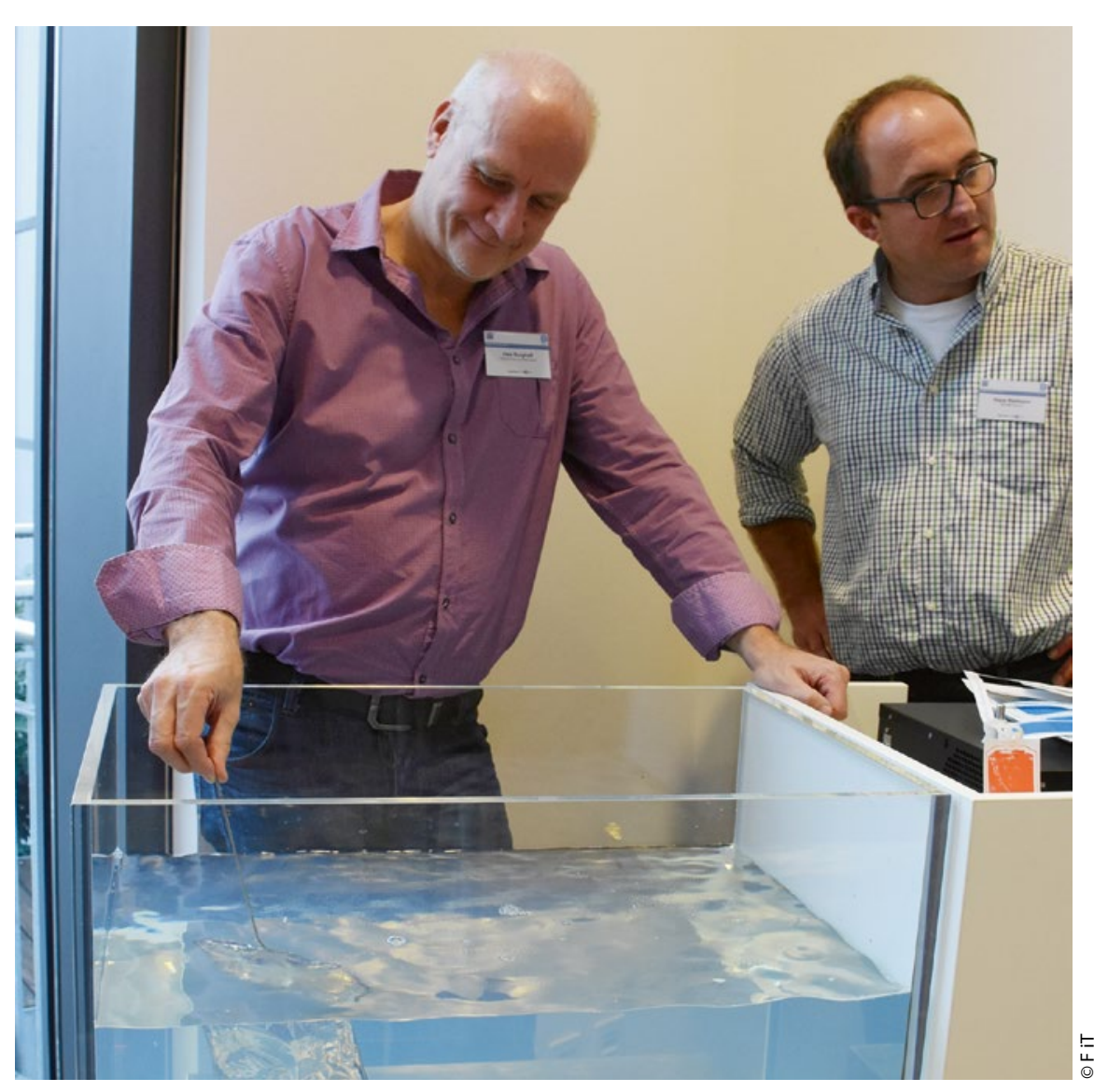

Auf der Agenda des Grundlagenseminars finden sich auch Vorträge und Praktika zur Wirkweise von Reinigungsmechanismen, hier beispielsweise zu Ultraschall. gen Sauberkeitsanforderungen bedarfsgerecht auszulegen beziehungsweise zu optimieren, zu steuern und zu überwachen. Im Mittelpunkt des Seminars „Prozessgestaltung“ am 20. Juni 2018 in Frankenthal stand grundlegendes Wissen zur Chemie des Reinigungsprozesses, Auswahl der Reinigungstechnik, Überwachung der Reinigungsmedien und der Sauberkeitskontrolle. Darüber hinaus erarbeiteten Anwendungstechniker mit den Teilnehmern in drei parallelen Workshops systematische Vorgehensweisen sowie praktikable und bewährte Lösungsansätze für diese Teilaufgaben. Die zweite Veranstaltung des Grundlagenseminars am 15. und 16. November 2018 in Frankenthal widmet sich der Prozessoptimierung. Am ersten Tag stehen zunächst Vorträge zu den Themen „Qualitätssicherung in der Bauteilreinigung“, „Chemie des Reinigungsprozesses“, „Überwachen der Reinigerkonzentration“ und „Kontrolle der Bauteilsauberkeit“ auf dem Programm. Diesem theoretischen Teil schließen sich parallele Praktika zu folgenden Themen an: „Prüfen der Bauteilsauberkeit auf filmische und partikuläre Verunreinigungen“, „Tensidkontrolle“ und „Builderkontrolle“.

Im Mittelpunkt des zweiten Tages stehen die Wirkweise von Reinigungsmechanismen sowie Lösungsansätze und Richtlinien für deren praktische und effiziente Nutzung. Schwerpunkte dabei bilden die Themen „Ultraschall“, „Chemie - emulgierend und demulgierend“ sowie „Konzipieren von Reinigungstechnik“.

Die Teilnehmerzahl ist auf jeweils 40 Personen begrenzt. //

Weitere Informationen: https://fit.zvo.org/ veranstaltungen/fit-termine.html fairXperts $\mathrm{GmbH}$ \& Co. KG Tel. 07025 8434-0 